\title{
An Intelligence-Based View of Firm Performance: Profiting from Artificial Intelligence
}

\author{
Ulrich Lichtenthaler \\ lichtenthaler@web.de | ISM - International School of Management, Im MediaPark 5c, 50670 Cologne, \\ Germany

\section{Letter from Academia}

\begin{abstract}
Many firms from diverse sectors increasingly use artificial intelligence (AI) to strengthen their efficiency by replacing human work. However, many of these advanced automation procedures will become standard tools in the future, and their relatively isolated application will not enable companies to gain a competitive advantage. Drawing on the distinction of data, information, knowledge and intelligence or wisdom, this paper suggests an intelligence-based view of firm performance as an extension of the knowledge-based view and the resource-based view. An intelligence-based perspective underscores the need to integrate AI applications with specific human expertise to complicate imitation by competitors. To sustain a competitive advantage over time, companies further need to develop a meta-intelligence to dynamically renew and recombine their intelligence architecture with multiple types of human and artificial intelligence. An intelligence-based view provides a systematic framework for assessing a firm's possibilities to profit from AI by generating growth and innovation beyond optimization and efficiency.
\end{abstract}

Keywords. Artificial Intelligence, Competitive Advantage, Digital Transformation, Firm Performance, Intelligence-based View, Knowledge-based View, Resource-based View.

Cite paper as: Lichtenthaler, U., (2019). An Intelligence-Based View of Firm Performance: Profiting from Artificial Intelligence - Letter from Academia, Journal of Innovation Management, www.open-jim.org, 7(1), 7-20. HANDLE: https://hdl.handle.net/10216/119830; DOI: https://doi.org/10.24840/2183-0606_007.001_0002 


\section{Introduction}

Many firms from diverse sectors increasingly apply artificial intelligence (AI) in terms of advanced data analytics and intelligent algorithms (Davenport \& Ronanki, 2018; Dubé, Du, McRae, Sharma, Jayaraman, \& Nie, 2018). Most of these firms focus on AI for replacing human work in selected processes (Agrawal, Gans, \& Goldfarb, 2018; Plastino \& Purdy, 2018). Examples include sectors as diverse as banking, e.g. Bank of America, and logistics companies like DHL. However, merely using AI to substitute human work is only a first step. These smart automation processes will become standard procedures, and they will not enable firms to achieve an intelligence-based competitive advantage in the future. Companies rather need to integrate AI with their human expertise (Lichtenthaler, 2018a). While it may be difficult for competitors to imitate human competencies, it is even more challenging to copy combinations of human and artificial intelligence.

If we take for granted the underlying economic logic of AI (Agrawal et al., 2018) and the need for a closer collaboration of humans and machines (Daugherty \& Wilson, 2018), companies still face the challenge of developing appropriate strategic initiatives to stay ahead of their competition. Many established strategy frameworks (Barney, 1991; Porter, 2008) allow for accommodating the recent developments of AI, but they do not help to fully map the competitive consequences of AI and its interplay with a firm's human expertise. In particular, the interrelationships of different intelligence types and their effect on firm performance may not be examined comprehensively with established strategy frameworks.

This paper aims at moving toward an intelligence-based view, which may provide some important contributions. First, it advances the established perspective of a knowledge-based view (Grant, 1996) by illustrating how different intelligence types help to leverage the underlying knowledge, information, and data. Second, the concept of intelligence architecture highlights the benefits of an integrated perspective on human and artificial intelligence (Daugherty \& Wilson, 2018). Third, an intelligence-based view provides new insights into the competitive effects of human expertise (Gardner, 1983). Fourth, it offers new insights into firms' possibilities to profit from AI (Davenport, 2018). Fifth and finally, an intelligence-based view is inherently dynamic, and this dynamic perspective enables the integration of different types of learning processes (Sirmon, Hitt, Arregle, \& Campbell, 2010).

\section{Extending the knowledge-based view}

A firm's ability to gain a competitive advantage at one point and to further sustain it over time is at the very heart of strategic management. Over the past decades, different frameworks have provided externally and internally driven views of understanding firm performance. Popular perspectives with a focus on external factors are the structure-conduct-performance and competitive forces frameworks (Bain, 1959; Porter, 1980). Here, the logic suggests that those firms will be successful which are competitively well positioned in an attractive industry. With regard to internal factors, the resource-based and competence-based perspectives have become very popular (Barney, 1991; Wernerfelt, 1984). On this basis, the dynamic capabilities and 
innovation-based perspectives put particular emphasis on the capabilities that are needed for dynamically sustaining a competitive advantage (Lichtenthaler, 2016; Rodrigues-Alves, 2018; Teece, Pisano, \& Shuen, 1997).

Along with a growing importance of knowledge management in the 1990s, the notion of a knowledge-based view of the firm was developed (Nonaka, 1994). It is an extension of the resource-based perspective because it considers knowledge as the key resource (Grant, 1996). Thus, firms may achieve a competitive advantage by developing particular knowledge from underlying information and data (Rowley, 2007). Specifically, data usually describes some discrete elements, such as numbers and words. If the data is organized, structured, and useful, it becomes information, which refers to linked elements, such as equations or sentences (Ili \& Lichtenthaler, 2017). If information gets meaning because it is contextualized, organized, and synthesized, it becomes knowledge, for example chapters or theories (Kogut \& Zander, 1992). There are other systematizations of data, information, and knowledge, but this understanding is consistent with the knowledge-based view (Rowley, 2007).

Basically, the knowledge-centered strategy framework suggests that the possibilities to achieve a competitive advantage from data and information are limited, whereas the step from information to knowledge is key because the synthesized and organized knowledge is complex and challenging to thoroughly understand and copy for competitors. Knowledge is often complex and difficult to imitate, especially tacit knowledge, which may not be articulated easily (Nonaka \& Krogh, 2009). Therefore, knowledge may provide an important basis for achieving a competitive advantage (Madhavan \& Grover, 1998). The knowledge-based view further enabled analyses how electronic knowledge management systems contribute to firm performance by helping to share information and knowledge within and between firms (Bock, Sabherwal, \& Qian, 2008). While the knowledge-based view has deepened our understanding of the role of knowledge and of knowledge management systems, it does not fully incorporate the impact of intelligence, neither human nor artificial intelligence.

Beyond gaining a competitive advantage due to different knowledge bases, firms may achieve superior performance because of a unique intelligence architecture, including their human and artificial intelligence. In this regard, intelligence may be regarded as the next step beyond knowledge which enables wisdom (Ili \& Lichtenthaler, 2017). Specifically, knowledge becomes intelligence if it is given insight and enables understanding because it is integrated and actionable (Rowley, 2007). Following the analogy of words, sentences, chapters - referring to data, information, and knowledge - an example for intelligence could be a book. Drawing on the analogy of numbers, equations, theories, an example for intelligence could be a system or paradigm. Thus, intelligence describes knowledge that is applied and organized to enable insights that could not be gained from knowledge without intelligence. As such, the intelligence-based view is an evolution of the knowledge-based view.

At first glance, the conceptual distinction of knowledge-based and intelligence-based perspectives may appear to be only academically relevant, but it also has major managerial implications. In particular, an intelligence-based view underscores that firms with the same knowledge may have significantly different performance outcomes. Even if two firms have access to the same internal and external knowledge, they may achieve different competitive positions if one firm 
has superior intelligence that enables specific insights as a basis for targeted competitive moves that the other firm lacks. These intelligence-based advantages may derive from human or artificial intelligence - or from a combination of the two. Independent of the particular type of intelligence, these performance differences may not be explained with a mere knowledge-based strategy framework.

For instance, imagine two firms becoming aware of new developments based on comprehensive technology foresight. Both companies gain some new knowledge about technological developments. The company with superior intelligence may generate valuable insights from the additional knowledge, and these insights may enable it to develop new products and services based on the technologies which open up a novel market. The competitive advantage in that market is primarily based on the firm's intelligence because the two companies had similar data, information and knowledge. Accordingly, this illustrative example shows a clear contribution of an intelligencebased view beyond knowledge-based arguments. Here, it is particularly important to note that this basic example does not require AI solutions to illustrate the value of an intelligence-based strategy framework.

These conceptual arguments help to clarify why different types of intelligence are competitively relevant beyond knowledge bases. To achieve a sustainable competitive advantage, it is therefore key for companies to develop intelligence that their existing and potential competitors lack. Despite the critical role of intelligence, however, an intelligence-based view of firm performance does not imply that data, information, and knowledge are not important. Rather, knowledge is also key in an intelligence-based context. If firms hypothetically only had intelligence without any underlying knowledge, they would continually develop similar types of knowledge again and again. A company with a high level of intelligence - human and/or artificial intelligence - would start unnecessarily reinventing different concepts, processes etc. without being able to achieve the benefits from the knowledge bases that its competitors probably have developed. Accordingly, developing high levels of intelligence without any underlying knowledge is not useful from a competitive point of view.

\section{Toward an intelligence-based view}

Intelligence can be defined in a variety of ways (Gardner, 1983; Hunt, 2011). Basically, it involves some mental activities, such as learning, reasoning, understanding, seeing relationships and more (Mueller \& Massaron, 2018). While algorithms and data analytics enable AI, no computer may entirely implement any of these mental activities so far (Mueller \& Massaron, 2018). Nonetheless, there have been major advances in different fields of AI in recent years. To profit from AI, it is therefore important to systematically understand what kinds of intelligence a human typically has and whether these mental activities may potentially be simulated in the foreseeable future. Ever since, human intelligence has provided a basis for competitive advantage (Daugherty \& Wilson, 2018). Some firms, such as Google and many consultancies, place emphasis on selecting candidates in some hiring processes based on selected indicators for different intelligence types. An intelligence-based view enables executives to highlight their employees' human intelligence as a particular competitive strength. 


\subsection{Human intelligence}

There are various reasons for assuming a positive performance impact of human intelligence (Mueller \& Massaron, 2018; Tegmark, 2018). In particular, it may have direct and indirect effects on firm performance. On the one hand, it may directly enable better results. For example, different human intelligences may help to take more informed managerial decisions, which positively affect subsequent performance. In a similar vein, particular engineering skills of human experts, for example at General Electric (Woyke, 2017), may contribute to solutions that are superior to competitors' products, leading to a competitive advantage that can quite easily be traced back to human expertise - even if intelligence leads to superior products only, which then provide the basis for enhanced performance. Beyond these relatively direct benefits, human intelligence may contribute to better decisions in selecting new employees, in wisely spending budgets, or in choosing the right partners for alliances or M\&A deals.

All of these key strategic variables are strongly affected by the intelligence of the humans that are involved. Thus, the overall impact of human intelligence goes far beyond some amazing ideas and extraordinary talents of selected employees. In particular, it also affects the choice of AI tools. Specifically, the effective use of AI depends on the managerial decision to rely on this type of AI for that particular application. Consequently, the impact of human intelligence on firm performance may be broader and stronger than you may initially assume, even if the growing relevance of $\mathrm{AI}$ is taken into account. While this impact may take many indirect forms and can go a relatively long chain of effects, it may often be traced back to particular types of human intelligence. There have been multiple attempts to systematize human intelligence, but the following classification of Howard Gardner has become a well-accepted overview. Specifically, the distinction is part of the theory of multiple intelligences, which divides human intelligence into various modalities rather than viewing it as one overall ability (Gardner, 1983). The theory distinguishes seven types of human intelligence, with different potential for simulation by means of computers (Gardner, 1983).

The first type of human intelligence is bodily-kinesthetic intelligence, which refers to the control of bodily motions and the ability to thoroughly handle objects (Gardner, 1983). This type of intelligence may be imitated by robots, even if they only performance repetitive tasks. The second type is creative intelligence, which refers to developing new patterns of thought that enable unique artistic, musical, or written output. The third type refers to interpersonal intelligence, which describes a human's sensitivity to others' feelings and the ability to collaborate in a group (Gardner, 1983). This type of intelligence is related to similar notions of emotional intelligence. The fourth type of human intelligence is intrapersonal intelligence, and it refers to inwardlooking and self-reflective capabilities. Thus, it enables a person to develop an understanding of one's strengths and weakness. As such, it currently is a human-only type of intelligence (Mueller \& Massaron, 2018). The fifth type is linguistic intelligence, and it describes a strong ability with words and languages. This type of intelligence includes, for example, understanding written and spoken input, processing it, and providing a useful and understandable answer. The sixth type is logical-mathematical intelligence, and it refers to logic, reasoning, numbers, and abstractions (Gardner, 1983). Thus, it enables calculating results, exploring patterns and 
drawing comparisons. Finally, the seventh type is visual-spatial intelligence, which refers to spatial judgment and visualization in physical environments (Gardner, 1983).

In addition to these seven types of human intelligence, which have been described by Gardner (1983), some other types have been suggested, including existential, naturalistic, and a teachingpedagogical intelligence. However, the focus here is on the original seven types because even adding several more will mostly likely not lead to an exhaustive list of intelligence types. From a management perspective, it further is not essential what particular segmentation of human intelligence is used. For achieving a competitive advantage, it rather is more important that a firm - or a firm's employees - have a particular ability and that the firm makes use of this ability. This application of human intelligence may occur in relative isolation or in combination with one or multiple types of AI.

\subsection{Artificial intelligence}

There are many different categorizations of AI, which generally refers to theory and computer systems that are able to perform tasks that usually would call for human intelligence (Mueller \& Massaron, 2018). In the field of what is considered AI today, machine learning has recently received most public attention (Agrawal et al., 2018; Finlay, 2017). Machine learning typically relies on algorithms and statistical techniques that enable computer systems to improve their performance in carrying out a particular task (Mueller \& Massaron, 2018). Machine learning relies on underlying training data, which enables the system to make predictions without being specifically programmed to complete the task. A further subset of machine learning is deep learning, which refers to learning data representations instead of focused and task-specific algorithms (Mueller \& Massaron, 2018).

Another important classification of AI distinguishes reactive machines, limited memory, theory of mind, and self-awareness (Mueller \& Massaron, 2018). Here, self-awareness describes systems that have an understanding of self and some consciousness. This would be the type of AI that is imagined in movies (Mueller \& Massaron, 2018; Wein, 2018). As such, this distinction is not central for competition in the near to medium future. Therefore, it may be more suitable to focus on different applications and use cases of AI (Davenport, 2018; Tegmark, 2018), even if they may not be mutually exclusive. For example, machine learning provides the basis for some applications that are considered as different categories. Nonetheless, the following distinction of seven types, which stems from integrating the categorizations in multiple books and encyclopedias, is helpful for discussing the competitive implications of AI (Daugherty \& Wilson, 2018; Davenport, 2018; Finlay, 2017; Ili \& Lichtenthaler, 2017; Mueller \& Massaron, 2018; Tegmark, 2018; Wein, 2018).

The first type of AI are expert systems, which are directed at imitating human decision-making. Typically, these systems comprise an underlying knowledge base with facts and rules as well as the inference engine which applies the rules (Jackson, 1998). The second type refers to machine learning, which includes for example deep learning and predictive analytics as core topics (Finlay, 2017). The third type involves various technologies that enable natural language processing. Here, the focus is on extracting, grouping, and categorizing information as well as 
translation and related applications (Millstein, 2018). The fourth type of AI describes planning and scheduling systems, which refer to the development of action strategies and sequences for subsequent execution. Typically, the emphasis is on complex solutions in a multidimensional space (Ghallab, Nau, \& Traverso, 2004). The fifth type refers to robotics and machines, and this field can be further segmented in reactive machines, limited memory, theory of mind, and selfawareness (Mueller \& Massaron, 2018). The sixth type of AI refers to speech synthesis systems, which include text-to-speech and speech-to-text solutions (Daugherty \& Wilson, 2018). Finally, the seventh type refers to computer vision, and it comprises image recognition, machine vision, and related applications (Daugherty \& Wilson, 2018). As mentioned above, these seven types constitute an integration of multiple existing segmentations of different types of AI.

\subsection{Human and artificial intelligence}

Applications of different types of AI may replace human work to some degree (Winick, 2018). However, a separate use of artificial and human intelligence is only one way for firms to profit from the recent advances in AI. In particular, replacing human work primarily enables firms to capture efficiency gains. While these benefits may have important positive effects on financial performance, they are one dimension of leveraging AI. If firms attempt to additionally develop novel solutions as a basis for further growth, they often need to combine one or multiple types of AI with one or multiple types of human intelligence (Lichtenthaler, 2018a). For example, enhanced algorithms for predicting customer behavior may be combined with human creativity to enable completely new business models. Thus, the augmentation of human intelligence needs to be distinguished from completely independent tasks that may be performed by AI (Daugherty \& Wilson, 2018). Beyond this example of an algorithm, you may also think about a surgical device which includes AI to enhance a surgeon's physical ability versus a robot which is able to make fully independent movements.

These interactions of artificial and human intelligence are at the core of the intelligence-based view (Figure 1). By developing and applying the different intelligence types - while considering their interdependencies - firms may gain a competitive advantage relative to other firms that lack similar intelligence levels. Artificial and human intelligence draw on the underlying data, information, and knowledge (Rowley, 2007). Without these underlying components, the value of most types of intelligence would be limited. Consider, for example, an algorithm for predictive maintenance that has been developed by General Electric. Without meaningful input data, even the most advanced version of this AI would provide limited value. In a similar vein, a human's interpersonal intelligence will provide limited benefits without further information. Accordingly, artificial and human intelligence build on the underlying knowledge base. At the same time, the development and application of the different intelligence types leads to the development of further data, information, and knowledge. Thus, a firm strengthens its knowledge by accumulating and enhancing its multiple intelligences.

Along with the underlying data, information, and knowledge, the combination of human and artificial intelligence may provide an important basis for competitive advantage (Lichtenthaler, 2018a). While a thorough understanding of these relationships may be essential for executives in the context of the recent advances in AI, the different intelligences only provide a first step (Da- 


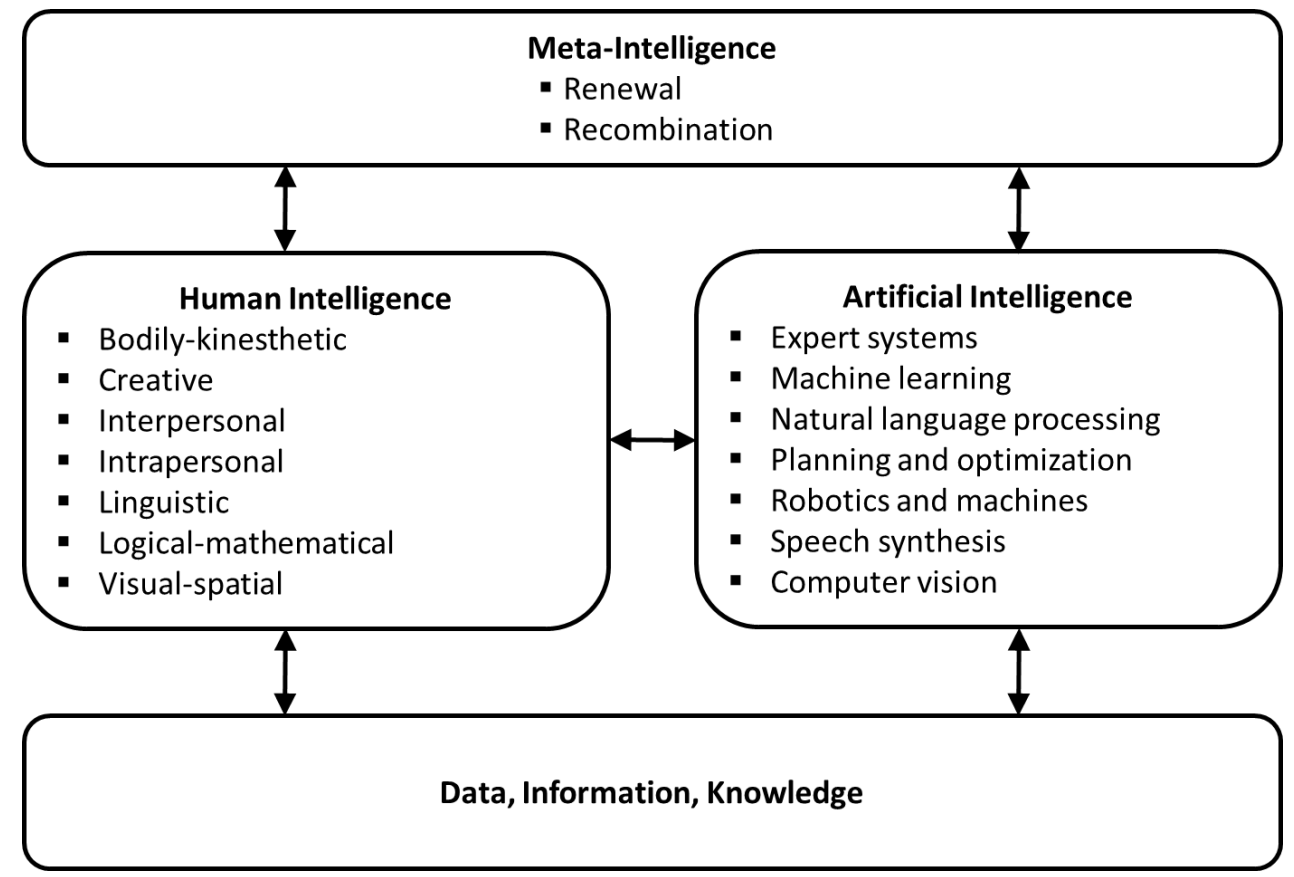

Fig. 1. Framework for an intelligence-based view.

venport, 2018). A firm's intelligence architecture may be excellent for achieving this competitive advantage today, but it will most probably be insufficient for keeping a strong competitive position in the future. Over time, a firm's intelligence-based advantage will inevitably deteriorate. With regard to human intelligence, for example, key persons may leave a firm, which needs to replace or ideally overcompensate for the different types of intelligence that are lost. Concerning AI, for example, a top position with regard to natural language processing today will not even be close to a top position in a few years because competitors quickly catch up and the evolution of this field is extremely dynamic.

As such, even the highest level of some intelligence will only enable a firm to achieve a temporary advantage, but it will often be insufficient for sustaining this advantage over time. Accordingly, firms do not only need to strengthen their different intelligence types. Instead, they also need to develop a meta-intelligence for dynamically transforming the different types of intelligence in alignment with their strategies. This meta-intelligence includes the renewal and recombination of the different intelligences (Lichtenthaler, 2019). Thus, it enables the targeted transformation of a firm's intelligence architecture to reactively respond or to proactively shape the changing competitive environment. Thus, firms need integrated intelligence - which consists of various types of artificial and human intelligence as well as the meta-intelligence (Lichtenthaler, 2019). This role of the meta-intelligence with regard to renewing and recombining a firm's multiple intelligences is particularly important for sustaining an intelligence-based competitive advantage. 


\section{Conclusion and outlook}

An intelligence-based view has some important implications. First, it substantially advances the established perspective of a knowledge-based view (Grant, 1996). In particular, it illustrates how different intelligence types may be developed and used for leveraging the underlying knowledge, information, and data (Rowley, 2007). As such, the focus on different types of intelligence further helps to understand major performance differences between firms with largely similar knowledge bases. Knowledge per se may not enable a firm to achieve a competitive advantage unless it is exploited in an intelligent way based on the business strategy. Thus, the multiple intelligences provide a critical missing link between a firm's knowledge base and subsequent performance outcomes.

Second, the concept of intelligence architecture highlights the need for an integrated perspective of the different intelligence types, which do not only build on the underlying knowledge, but also contribute to its extension and to the generation of completely new knowledge (Rowley, 2007). In particular, this understanding of a company's intelligence architecture highlights the need for going beyond isolated AI applications. If executives aim at fully leveraging the benefits of AI, they need to put the interfaces between AI and the remaining part of the intelligence architecture at the core of their attention (Lichtenthaler, 2019). These interfaces will be critical for achieving a competitive advantage because they may not be imitated as easily as stand-alone AI solutions, whose potential impact on gaining a competitive advantage may decrease in the future because they will be applied by many competitors. In contrast, a firm's complex intelligence architecture will continue to provide a sound basis for a sustainable competitive advantage.

Third, an intelligence-based view offers new insights into the competitive effects of human intelligence (Gardner, 1983). While superior human resources play a major role in other perspectives on firm performance, such as the resource-based view and knowledge-based view (Barney, 1991; Nonaka, 1994), an intelligence focus offers a more thorough understanding how the different types of human intelligence may contribute to achieving a competitive advantage. In particular, the focus on multiple human intelligences (Gardner, 1983) enables systematic analyses of interdependencies with AI. In this regard, it is particularly important to underscore the core role of human expertise despite the substantial advances of AI. There are manifold reasons for the continuing importance of human experts who, for example, select AI applications in companies. Even if AI further advances in the future, it will substantially depend on human expertise and intelligence to unfold a competitive impact.

Fourth, an intelligence-based perspective provides a systematic framework for exploring a firm's possibilities to profit from AI (Davenport, 2018). In particular, it helps to examine the direct and indirect performance effects of different types of AI. As such, it emphasizes the need for analyzing the interplay of AI with various types of human intelligence (Lichtenthaler, 2018a). A specific AI application may strengthen a competitive advantage if it goes along with a suitable type of human intelligence. In contrast, the same AI may provide hardly any benefit if it is not aligned with the remaining parts of a firm's intelligence architecture. This key role of the organizational context of using AI has been overlooked quite often. In many situations, AI does not automatically contribute to performance. Instead, strategic alignment is core for ensuring that the investments in AI pay off. The importance of a firm's intelligence architecture will 
further grow because particular AI applications will increasingly become standard tools, which many firms may use with nearly identical proficiency.

Fifth and finally, an intelligence-based view is inherently dynamic which enables the integration of individual and organizational learning processes (Argote, 1999; Sirmon et al., 2010). The recent technological advancements emphasize the need for staying ahead of competition in terms of developing, customizing, and applying the latest AI. In light of the dynamics of many firms' competitive environments, the continuous development and extension of human intelligence becomes even more critical than in the past. An intelligence-based view enables a comprehensive perspective on a firm's intelligence architecture. Here, the different types of artificial and human intelligence provide immediate starting points for adapting and transforming established competencies. Specifically, the concept of a meta-intelligence highlights the need for an ongoing transformation of the intelligence architecture (Lichtenthaler, 2019). Without renewing the different intelligence types, a competitive advantage will be lost in a relatively short time. Besides renewing the intelligences, executives need to recombine the interrelationships among the different intelligence types to ensure that the interfaces continue to contribute to a firm's future competitive position.

AI has come a long way, but for most companies the largest part of the transformation is still ahead. Executives need to acknowledge that many changes cannot fully be foreseen today. Thus, some level of experimentation and simultaneously pursuing multiple options will be inevitable (Bühring \& Moore, 2018; Lichtenthaler, 2018b; Wilbert, Durst, Ferenhof, \& Selig, 2018). Specific attention needs to be put on the dynamics of competition with regard to new activities in the value chain, such as data analytics, and new players in the competitive ecosystem, such as IT service providers (Brito, 2018; Ili \& Lichtenthaler, 2017). Acknowledging this strategic complexity and developing a systematic strategic approach is essential for achieving a competitive advantage (Apanesevic, Arvidsson, \& Markendahl, 2018; Datta, 2018). While the growing competitive relevance of AI cannot be ignored, the core strategic impact will derive from the interplay of AI with human intelligence rather than from the pure implementation of AI. In this respect, an intelligence-based understanding of future competitive advantage is an important starting point.

\section{References}

Agrawal, A., Gans, J., \& Goldfarb, A. 2018. Prediction machines: The simple economics of artificial intelligence. Boston: Harvard Business Review Press.

Apanesevic, T., Arvidsson, N., \& Markendahl, J. 2018. Mobile payments: a proposal for a context-oriented approach based on socio-technical system theory. Journal of Innovation Management, 6(3): 40-97.

Argote, L. 1999. Organizational Learning: Creating, Retaining and Transferring Knowledge. Boston: Kluwer.

Bain, J. S. 1959. Industrial Organization. New York: Wiley. 
Barney, J. 1991. Firm Resources and Sustained Competitive Advantage. Journal of Management, 17: $99-120$.

Bock, G.-W., Sabherwal, R., \& Qian, Z. 2008. The Effect of Social Context on the Success of Knowledge Repository Systems. IEEE Transactions on Engineering Management, 55: $536-551$.

Brito, C. 2018. Promoting the creation of Innovation Ecosystems: the case of the University of Porto. Journal of Innovation Management, 6(3): 8-16.

Bühring, J., \& Moore, P. A. 2018. Emotional and Social Intelligence as 'Magic Key' in Innovation: A Designer's call toward inclusivity for all. Journal of Innovation Management, 6(2): 6-12.

Datta, S.P.A. 2018. Unleashing the new Wealth Of Nations. Journal of Innovation Management, 6(2): 13-37.

Daugherty, P. R., \& Wilson, H. J. 2018. Human + machine: Reimagining work in the age of AI. Boston: Harvard Business Review Press.

Davenport, T. H. 2018. AI Advantage: How to Put the Artificial Intelligence Revolution to Work. Cambridge: MIT Press.

Davenport, T. H., \& Ronanki, R. 2018. Artificial Intelligence for the Real World. Harvard Business Review, 96: 108-116.

Dubé, L., Du, P., McRae, C., Sharma, N., Jayaraman, S., \& Nie, J.-Y. 2018. Convergent Innovation in Food through Big Data and Artificial Intelligence for Societal-Scale Inclusive Growth. Technology Innovation Management Review, 8(2): 49-65.

Finlay, S. 2017. Artificial Intelligence and Machine Learning for Business: A No-Nonsense Guide to Data Driven Technologies. Preston: Relativistic.

Gardner, H. K. 1983. Frames of mind: The theory of multiple intelligences. New York: Basic Books.

Ghallab, M., Nau, D., \& Traverso, P. 2004. Automated planning: theory and practice. Amsterdam: Elsevier.

Grant, R. M. 1996. Toward a knowledge-based theory of the firm. Strategic Management Journal, 17, Winter Special Issue: 109-122.

Hunt, E. 2011. Human Intelligence. Cambridge: Cambridge University Press.

Ili, S., \& Lichtenthaler, U. 2017. FAQ Digital Transformation and Artificial Intelligence: 101 Questions - 101 Answers. Karlsruhe: Ili Consulting.

Jackson, P. 1998. Introduction to expert systems. Harlow: Addison-Wesley.

Kogut, B., \& Zander, U. 1992. Knowledge of the firm, combinative capabilities, and the replication of technology. Organization Science, 3: 383-397.

Lichtenthaler, U. 2016. Toward an innovation-based perspective on company performance. Management Decision, 54: 66-87. 
Lichtenthaler, U. 2018a. Substitute or synthesis? The interplay between human and artificial intelligence. Research-Technology Management, 61(5): 12-14.

Lichtenthaler, U. 2018b. The world's most innovative companies: a meta-ranking. Journal of Strategy and Management, 11(4): 497-511.

Lichtenthaler, U. 2019. Beyond artificial intelligence: why companies need to go the extra step. Journal of Business Strategy, 39.

Madhavan, R., \& Grover, R. 1998. From Embedded Knowledge to Embodied Knowledge: New Product Development as Knowledge Management. Journal of Marketing, 62: 1-12.

Millstein, F. 2018. Natural language processing with Python. London: CreateSpace.

Mueller, J. P., \& Massaron, L. 2018. Artificial Intelligence for Dummies. Hoboken: Wiley.

Nonaka, I. 1994. A Dynamic Theory of Organizational Knowledge Creation. Organization Science, 5: 14-37.

Nonaka, I., \& Krogh, G. von. 2009. Tacit Knowledge and Knowledge Conversion: Controversy and Advancement in Organizational Knowledge Creation Theory. Organization Science, 20: 635-652.

Plastino, E., \& Purdy, M. 2018. Game changing value from Artificial Intelligence: eight strategies. Strategy \& Leadership, 46: 16-22.

Porter, M. E. 1980. Competitive Strategy. New York: Free Press.

Porter, M. E. 2008. The five competitive forces that shape strategy. Harvard Business Review, 86: 79-93.

Rodrigues-Alves, M. F. 2018. Literature on organizational innovation: past and future. Innovation \& Management Review, 15(1): 2-19.

Rowley, J. 2007. The wisdom hierarchy: representations of the DIKW hierarchy. Journal of Information and Communication Science, 33(2): 163-180.

Sirmon, D. G., Hitt, M. A., Arregle, J.-L., \& Campbell, J. T. 2010. The dynamic interplay of capability strengths and weaknesses: Investigating the bases of temporary competitive advantage. Strategic Management Journal, 31: 1386-1409.

Teece, D. J., Pisano, G., \& Shuen, A. 1997. Dynamic capabilities and strategic management. Strategic Management Journal, 18: 509-533.

Tegmark, M. 2018. Life 3.0: Being human in the age of artificial intelligence. New York: Vintage Books.

Wein, E. 2018. Artificial Intelligence Making Machines Learn: A friendly Introduction.

Wernerfelt, B. 1984. A Resource-based View of the Firm. Strategic Management Journal, 5: $171-180$.

Wilbert, J.K.W., Durst, S., Ferenhof, H. A., \& Selig, P. M. 2018. Unlearning at the Individual Level: An Exploratory Case Study in a High Power Distance Country. Journal of Innovation Management, 6(3): 17-39. 
Winick, E. 2018. Every study we could find on what automation will do to jobs, in one chart. MIT Technology Review, 01/25/2018.

Woyke, E. 2017. General Electric builds an AI workforce. MIT Technology Review, Online: $1-3$. 


\section{Biographies}

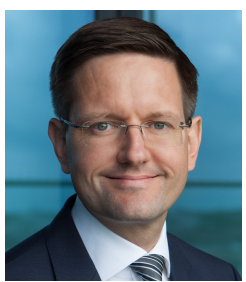

Ulrich Lichtenthaler. Ulrich Lichtenthaler is a Professor of Management and Entrepreneurship at ISM - International School of Management in Cologne, Germany. He holds a Ph.D. degree in technology management and further is an Executive Consultant, who has successfully completed over 20 digital transformation projects over the past years. He has written multiple books and articles for journals and newspapers, such as MIT Sloan Management Review and Wall Street Journal, and he has taught executive education courses at leading business schools. 